\title{
La construcción de una exposición de arte contemporáneo: Cooperación de actores y dilemas en un montaje del Museo de Artes Visuales
}

\author{
The construction of a contemporary art exhibition: Cooperation and dilemmas \\ in the Museum of Visual Arts, Chile
}

Francisca Ortiz Ruiz Centro de Investigaciones Socioculturales CISOC, Universidad Alberto Hurtado, Chile. Contacto: franortizruiz@gmail.com

\begin{abstract}
Resumen
La finalidad del artículo es problematizar el proceso de gestación de la obra de arte, develando las prácticas invisibilizadas en la construcción de una exposición de arte contemporáneo. Se realizó una aproximación etnográfica al interior del montaje de la exposición "Nuestro sitio" del Museo de Arte Visuales en Chile. Develando con ello, la conformación de una exposición en un museo, la noción de montaje, la consideración de las redes que la componen y la emergencia de dilemas que influyen en la obra de arte. Concluyendo que la gestación de la obra de arte contemporáneo es un proceso que emerge cuando estéticamente se presenta el resultado de un trabajo cooperativo de variados actores en un espacio específico y por distinciones propias del lenguaje artístico.
\end{abstract}

Palabras claves: Museo, Arte, Exposición, Redes, Obra de arte.

\begin{abstract}
This article problematizes the gestation of a work of art, exposing the invisible practices in developing a contemporary art exhibition. Data is based on an ethnographic study of the installation of the exhibition "Our Site" at the Museum of Visual Arts in Chile. The article reveals the process of establishing an exhibition in a museum and the notion of montage, and considers the composition of networks and the emergence of dilemmas which influence a work of art. I conclude that the gestation of a contemporary artwork is a process that emerges aesthetically when the result of a collaborative work of various actors is presented in a specific space, and by distinction in its own artistic language.
\end{abstract}

Keywords: Museum, Art, Exhibition, Networks, Artwork.

Francisca Ortiz Ruiz,

La construcción de una exposición de arte contemporáneo: Cooperación de actores y dilemas en un montaje del Museo de Artes Visuales, Autoctonía. Revista de Ciencias Sociales e Historia, Vol. I, №2, Julio-Diciembre 2017, 254-268

ISSN 0719-8213 


\section{Introducción}

Saliendo de la estación de metro Universidad Católica en Santiago de Chile, caminando por pequeñas calles de data antigua, pasando por una gran cantidad de turistas, de negocios, restaurantes y librerías, se llega a la Plaza Mulato Gil de Castro: Un callejón, un poco escondido de los ruidos de la ciudad, y que al final a la derecha tiene unas puertas de vidrio. $\mathrm{Al}$ atravesarlas, se entra a otro mundo, a un lugar donde las formas cobran otros sentidos, y donde la arquitectura juega con los transeúntes. Es un gran cubo blanco con suelos de madera, y que en sus muros lleva una gran cantidad de objetos que salen de sus contextos cotidianos, para ingresar a este recinto en algo titulado 'exposición'. Éste es el Museo de Artes Visuales (MAVI).

¿Cómo fue que llegó a existir esta exposición? ¿Qué pasó que de un momento a otro se nombran a una gran cantidad de objetos distintos como 'obras de arte'? ¿Cuándo y cómo se gesta realmente está obra de arte? ¿Cuál es la caja negra que se invisibiliza dentro de este cubo blanco? Estas son solo pocas de las interrogantes que pueden surgir de este escenario.

Por medio de un estudio de caso realizado entre los meses de agosto y noviembre del año 2012, se busca esclarecer la 'caja negra' que es invisibilizada en el 'cubo blanco'. Más específicamente, se presentan los resultados obtenidos a partir de una etnografía de seis días al interior del montaje de la exposición "Nuestro sitio", en el Museo de Artes Visuales.

En los montajes de una exposición, hay controversias y en la mayoría de los casos, actores no humanos fueron elementos centrales en estas diversas discusiones. Los objetos, son reales mediadores de la acción, y finalmente de lo que se conoce como la obra de arte expuesta en el museo. Los distintos actores humanos, develan sus variadas visiones: un maestro, ve la utilidad del objeto; la curadora, intenta mantener el sentido de la obra en el espacio; la productora se preocupa de los recursos disponibles y de la concretización del montaje; y por último, el artista observa si la obra funciona o no de la forma en que era montada.

La gestación de la obra de arte ha sido una disyuntiva a tratar a lo largo de la sociología del arte en general (Heinich, 2002: 10). Es en esta discusión, que este artículo pretende aportar tanto empírica, como teóricamente, con observaciones realizadas sobre la relevancia del: (1) espacio y de los actores (tanto humanos como no humanos), (2) la cooperación entre varios actuantes mediante prácticas, acciones y conocimientos, y (3) todas los dilemas que se fueron tomando a lo largo del montaje físico de la exposición: cada clavo y cada centímetro fueron centrales en la construcción de "Nuestro sitio".

Según Howard Becker una exposición, se construye en un mundo del arte (2005: 17). Allí se coordinan distintos grupos de personas que, por medio de vínculos cooperativos generan lo que conocemos como obra de arte. "El artista, entonces, trabaja en el centro de una red de personas que colaboran, cuyo trabajo es esencial para el resultado final. Dondequiera que el artista dependa de otros existe un vínculo cooperativo" (Becker, 2005: 43). Un vínculo que, como veremos más tarde, se visibiliza en el montaje de la exposición de sus obras. Proceso que es invisibilizado al público por este gran cubo blanco que es el museo.

La variedad de actores y de operaciones (por pequeñas que sean) envueltos en la instalación de una exposición, son relevantes al momento de generar una obra de arte (Yaneva, 2003: 126). Cada detalle en el montaje es importante. Sobre todo en el arte contemporáneo, entendidos como pulsos con distintos ritmos en su transformación (Yaneva, 2003: 169). 'Actores permanentes' y 'temporales', 'humanos' y 'no humanos', que además de influir como diría la autora, cooperan en la construcción de una obra.

Domínguez el año 2012 por su parte, mediante un estudio de la elaboración de la instalación Spiral Jetty, confirma la idea anterior. Por medio de detalladas y meticulosas descripciones del proceso, el autor logra abrir la caja negra que devela la gestación misma de la obra. Para su comprensión, desde las decisiones que van siendo tomadas, paso a paso, hasta los materiales de construcción son claves. Ya que se logra por medio de que se asocian todo tipo de actantes en la producción. ¿Pero realmente cómo se llega a tener una obra presente en un museo? ¿Qué pasa en su montaje? ¿Hay actantes con vínculos cooperativos? ¿Quiénes son ellos? Volvemos entonces, a la pregunta por la caja negra del cubo blanco. 


\section{El caso: Exposición "Nuestro sitio".}

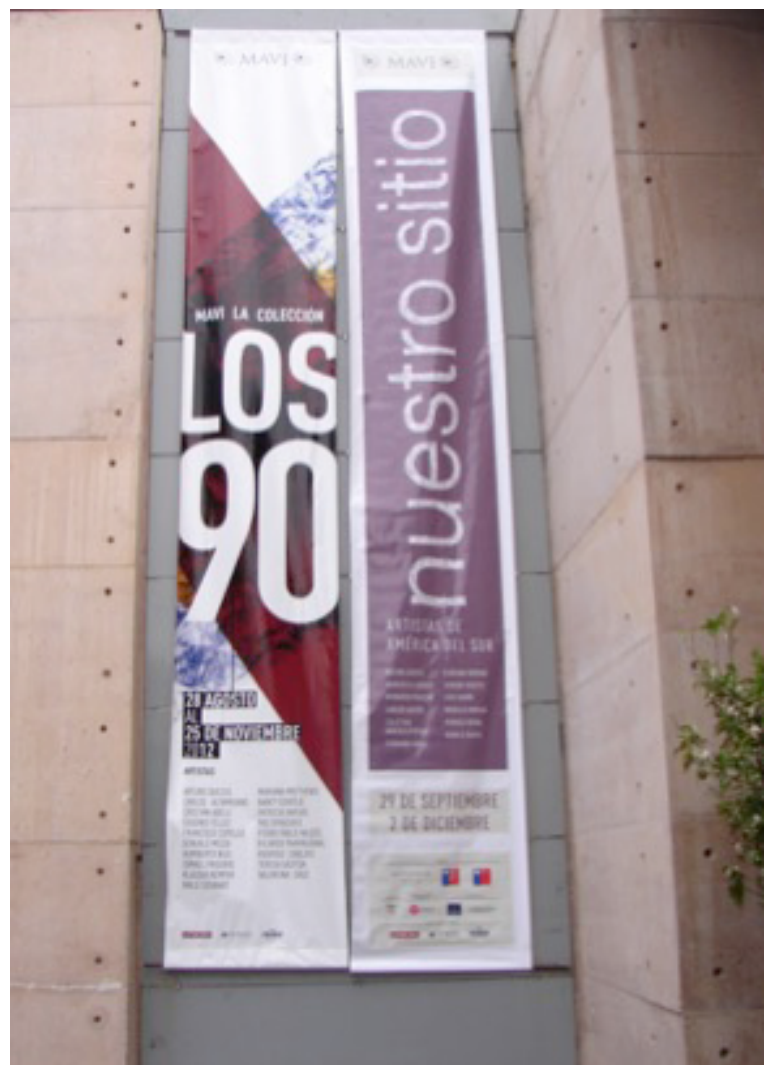

Lienzos fuera del MAVI mostrando a los transeúntes, lo que se expone en el museo

Desde el viernes 28 de septiembre del año 2012, al domingo 2 de diciembre del mismo año, se realizó en el Museo de Artes Visuales (MAVI) de Chile, la exposición "Nuestro sitio". Esta iniciativa parte desde la curadora chilena Beatriz Bustos, en asociación con el Museo de Arte Contemporáneo (MAC) de Niteroi, Brasil. Para luego ver en el MAVI un lugar en Chile, para exponer "Nuestro sitio".

Nueve artistas de distintos países participan de esta muestra, que gira en torno a tres ejes de dinámicas, presentes en la región latinoamericana: Consumo/ mercado, ideología/tensiones y deslindes/ migraciones. El objetivo es generar una reflexión desde la mirada del arte, sobre lo que acontece actualmente en el continente latinoamericano[. En un primermomento, la exposición fue presentada en el MAC de Niteroi, Brasil, desde el 5 de mayo al 24 de junio de este año. Los artistas presentes son: (A) de Chile: Livia Marín, Bernardo Oyarzún, Marcela Moraga, Rodrigo Bruna; (B) de Colombia: Barbarita Cardozo, Fernando Arias, Carlos Castro; (C) Bolivia: Joaquín Sánchez; (D) de Ecuador: Avelina Crespo; (E) y finalmente desde Brasil: Ronald Duarte,
Floriano Romano y el colectivo Gráfica Utópica. En la exposición se reúnen instalaciones, fotografías, acuarelas, videos, esculturas y performance.

La exposición cuenta con el financiamiento de organizaciones de distintos países, pero cuando fue traída a Chile, los principales auspiciadores fueron FONDART (Fondo Nacional de Desarrollo de las Artes y la Cultura) y el DIRAC (Dirección de Asuntos Culturales del Ministerio de Relaciones Exteriores). Mientras que las dos instituciones a cargo de su producción ejecutiva eran el Museo de Artes Visuales y la Fundación Mulato Gil de Castro, quienes prestaron sus dependencias para ello. Espacio en el cual fue desarrollada la observación etnográfica del montaje realizado en la exposición de este museo.

\section{3. ¿Cómo investigar el montaje en un museo?}

Entre los días lunes 24 (fecha en la que se empieza el montaje de la exposición en cuestión) y el viernes 28 de Septiembre (con la inauguración), se llevó a cabo una aproximación etnográfica (Guber, 2001: 65) con las debidas preocupaciones éticas que el contexto demandaba (Wolcott, 2006: 292). Siguiendo el ejemplo de Bruno Latour, en el caso de Aramis (1994: 77) o en varios de sus estudios en terreno (2001: 38), esta técnica fue escogida principalmente por tres razones: (1) Por ser un enfoque que estudia el escenario, en sus contextos naturales, "porque todo aquello que está relacionado con, o contenido en, o que depende de (son muchas las opciones) la ubicación geográfica necesariamente afecta lo que estamos estudiando" (Becker, 2011: 78). (2) Por que el investigador ejerce una influencia mínima en el campo que busca estudiar, generando un conocimiento que es de carácter inductivo y flexible. (3) Usando la etnografía, vemos una realidad donde "el propio mundo está articulado, y no al revés” (Latour, 2001: 355), es debido a su carácter relacional, procesual y situado que la etnografía funciona. Generando la comprensión de los eventos, los procesos y el desarrollo del montaje en sí mismo (tanto en las redes que convoca, como en los dilemas que conlleva).

La etnografía fue de "corte transversal" (ÁlvarezGayou, 2009: 77), ya que fue en un momento determinado en el tiempo. Se desarrolló desde el término del desmontaje de la exposición anterior, hasta la inauguración de la exposición "Nuestro sitio". La observación principal se centró en el transcurso del montaje, incluyendo también el conversatorio 
(realizado una hora antes de la inauguración) entre los artistas que exponían.

Se colocó especial énfasis en lo relacional (Law, 2007: 150), en la constante relación de elementos que es performativa (Ramos, 2012: 236). Es decir, donde no se busca entender a los objetos como fijos, sino que como performativos y móviles. Los objetos así, en su relación con los actores humanos, están inmersos en una red que con el uso de la etnografía se plasma más avanzado el artículo.

El registro de las observaciones realizadas, fueron realizadas todos los días, luego de cada ida al lugar de exposición. Dado que los datos y la información recopilada abarcaban todo el montaje de dicha muestra, se consideró que esta era suficiente para realizar un análisis del tema de investigación debido al logro de una descripción del escenario vivenciado lo suficientemente denso (Geertz, 1992: 32), como para generar una mejor comprensión de ello. Se considera por densidad en estas observaciones, en los casos en que la sola descripción profunda de estos eventos, genera una imagen lo suficientemente abarcadora y detallista, como para poder revivir los hechos que fueron registrados por la investigadora. Esta información se enriqueció más aún al integrar la fotografía (Banks, 2010: 20) en la captura de todos los días de la observación, transformandose en un recurso central de recolección de datos y registro audiovisual (Hammersley et. al, 2001: 10).

\section{Resultados}

\subsection{El transcurso de las 'labores cooperativas' en un museo}

"Nuestro sitio" es un transcurso de varios procesos, que se caracterizan principalmente por su componente cooperativo. Un museo al enfrentarse a la tarea de generar una exposición, no es solo una o dos etapas solamente: son varias. Todas estas 'labores cooperativas' que llamo, no es lineal. Se van sobreponiendo entre ellas, y nunca de igual forma. Son transiciones en un transcurso de tiempo, que forman las exposiciones en un museo.

Son conceptualizadas como labores cooperativas, en referencia a la noción de "vínculos cooperativos" de Howard Becker (2005: 54). Para él estos son parte de los mundos del arte, y sus actividades son necesarias para la producción artística y la continuación de ciertas convenciones (Becker, 2005: 55). Pero en este artículo, se considera que varios de estos vínculos se diferencian y otros se parecen, entre sí. Es por ello que una labor cooperativa, se entiende que posee varios vínculos cooperativos. Pero generalmente son observadas desde lo que visibilizan: las exposiciones, y la inauguración de éstas. Pero suceden más eventos en un museo. A continuación menciono los principales eventos, que llamo las 'labores cooperativas':

Esquema $n^{\circ} 1$ : 'Labores cooperativas' en un museo.

Elaboración propia, a partir de información obtenida de la etnografía (septiembre y octubre, 2012) en MAVI, Chile.

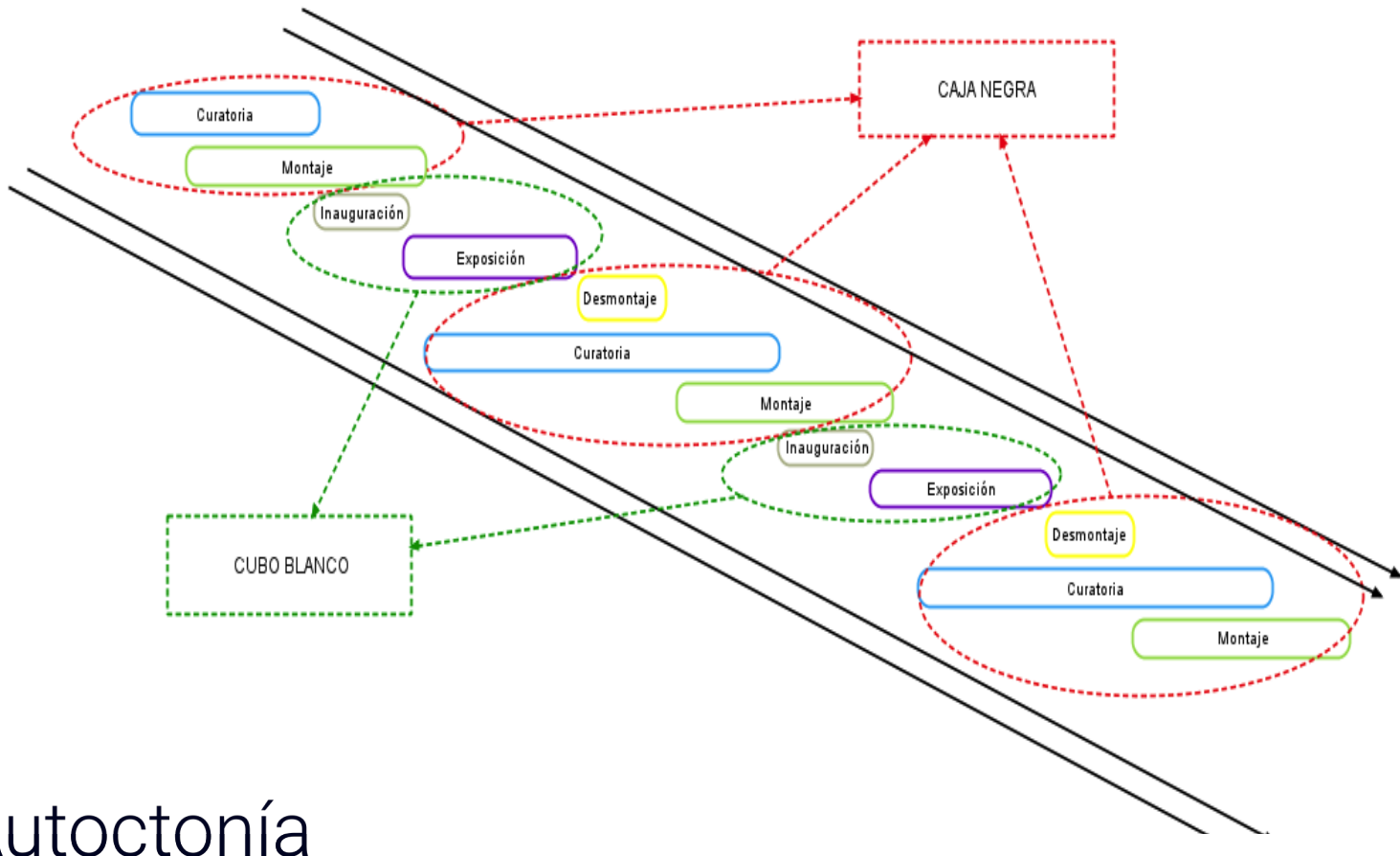


Lo que se presenta en el esquema es lo siguiente: un transcurso de tiempo, que no es lineal, ocurriendo en un museo. Allí conviven distintas etapas o procesos o 'labores cooperativas', que marcan la comprensión de lo que realmente construye este tipo de organizaciones. Cada una de éstas, se encuentra con un color diferenciado, para demarcar la diferencia entre ellos. Aún así todos están dentro de la misma historia, al encontrarse al interior de las flechas negras que lo rodean. Estas labores, como ya se mencionó, se sobreponen dependiendo de lo que sucede en aquel momento en el museo.

El MAVI es un lugar que posee seis salas disponibles para realizar exposiciones. Es común, considerando este espacio, la posibilidad de que al generar varias exposiciones a la vez, se sobreponen en el tiempo. Esto siempre es según la contingencia del programa expositivo decidido por el museo. A veces el montaje de una exposición calza con el desmontaje de otra o con la curatoría.

Ahora bien, están las líneas puntuadas, que estipulan una división entre las labores cooperativas en dos, surgiendo así, el 'cubo blanco' y la 'caja negra'. El primero hace referencia a los eventos públicos que son visibles por todos los asistentes al museo, tanto del interior, como del exterior de la organización. Es lo que se puede mostrar a personas externas, es decir, las exposiciones ya montadas y la inauguración de estas mismas, incluyendo también actividades educacionales que son realizadas a partir de las exposiciones, en el mismo lugar.

Por otro lado, está también la denominación de la 'caja negra'. Aludiendo a lo privado del museo, lo que ocurre que debe ser invisible a los ojos de los visitantes y solo es conocido por quienes componen al museo. Erving Goffman, señala una diferencia entre lo que significa estar en la parte visible del escenario, en la obra misma, y lo que es el trasfondo del escenario. Lo visible es este cubo blanco, que hace alusión al museo que se presenta al público, sin mayores tensiones o problemáticas por resolver. Mientras que el trasfondo (la caja negra) es donde opera la utilería y la fachada, es "un lugar, relativo a una actuación determinada, en el cual la impresión fomentada por la actuación es contradicha a sabiendas como algo natural" (Goffman, 2004: 123). Es trazar una diferencia entre lo que se muestra y lo que no, el frontis del escenario y el trasfondo, lo visible y lo invisible. Esta caja que representa lo invisible, se encuentra compuesta por tres subdivisiones: el desmontaje, la curatoría y el montaje.
El primero, generalmente dura entre dos y cuatro días, siendo el momento en el cual retiran todas las obras ya expuestas. El segundo, es paralelo a muchas otras labores (conversación con los artistas, negociaciones, organización administrativa, etc.), durando mucho más tiempo, ya que es un trabajo más individualizado e impredecible de parte del curador con las obras (antes, durante y después de la exposición). Por último, está el montaje, el momento en que se colocan las obras de arte, a partir de la cooperación entre distintos actantes. El montaje así es el proceso mediante el que se le da forma a la exposición en las salas del museo.

Pareciera ser entonces que el montaje es uno de los momentos claves en la concepción de las obras en un espacio en particular. Ciertas visiones verían las obras de arte, principalmente no como un producto del artista (Bourdieu, 2010: 65), sino que del campo social (Bourdieu, 2002: 122). Mientras que otras, mencionan que "el significado de una obras de arte o exposición surge a través de la artista, el curador, y otro técnico, con créditos (que no son necesariamente conscientes) de los objetos y el espacio." (Acord, 2010: 462). En tanto en el presente documento, el análisis se centra en la gestación de la obra en el montaje propio de una exposición de arte contemporáneo.

\subsection{Los actores en la construcción de "Nuestro sitio"}

$\mathrm{Al}$ entrar a un museo, generalmente vemos las mismas cosas: Una recepción, espacios con exposiciones perfectamente instaladas y algunos humanos que recorren el lugar. ¿Pero siempre es así? No. Lo que aquí se plantea, es justamente eso. Hacer visible lo invisible, y por sobre todo en el proceso de montaje de estas obras. En la misma línea, el museo se entiende como un espacio que se visualiza "siempre perfecto ante los que vienen"2 a observar las exposiciones. Es justamente en lo que no se ve, en lo que está oculto bajo la idea de museo, lo que se indago por medio de la etnografía. La recopilación de ésta información de lo invisible en un museo se retrata en el 'Esquema n ${ }^{\circ}$ ': el montaje de la exposición'.

Son diferentes actantes, que en su relación con los actores humanos, van construyendo lo que luego conocemos como una exposición. Para describir este proceso, se desarrolló la construcción del 'Esquema 
n 2: Actores de la exposición "Nuestro sitio". Se compone de la red de actores que influenciaron, de alguna u otra forma en la concepción de esta exposición. Sus vinculaciones son de carácter 'dependiente' (Dep) o 'codependiente' (Co-Dep); en el caso de la primera, es cuando un actor depende en su actuar de otro actor; mientras que en el segundo, es más bien una dependencia mutua entre ambos actores que están unidos. Comprendiendo el modo de relación que se simboliza, pasamos a los momentos de la exposición que en el esquema, se representan por dos recuadros grandes que encierran en ellos distintos actores y relaciones. Son tres:

\section{Esquema n²: Actores de la exposición "Nuestro sitio".}

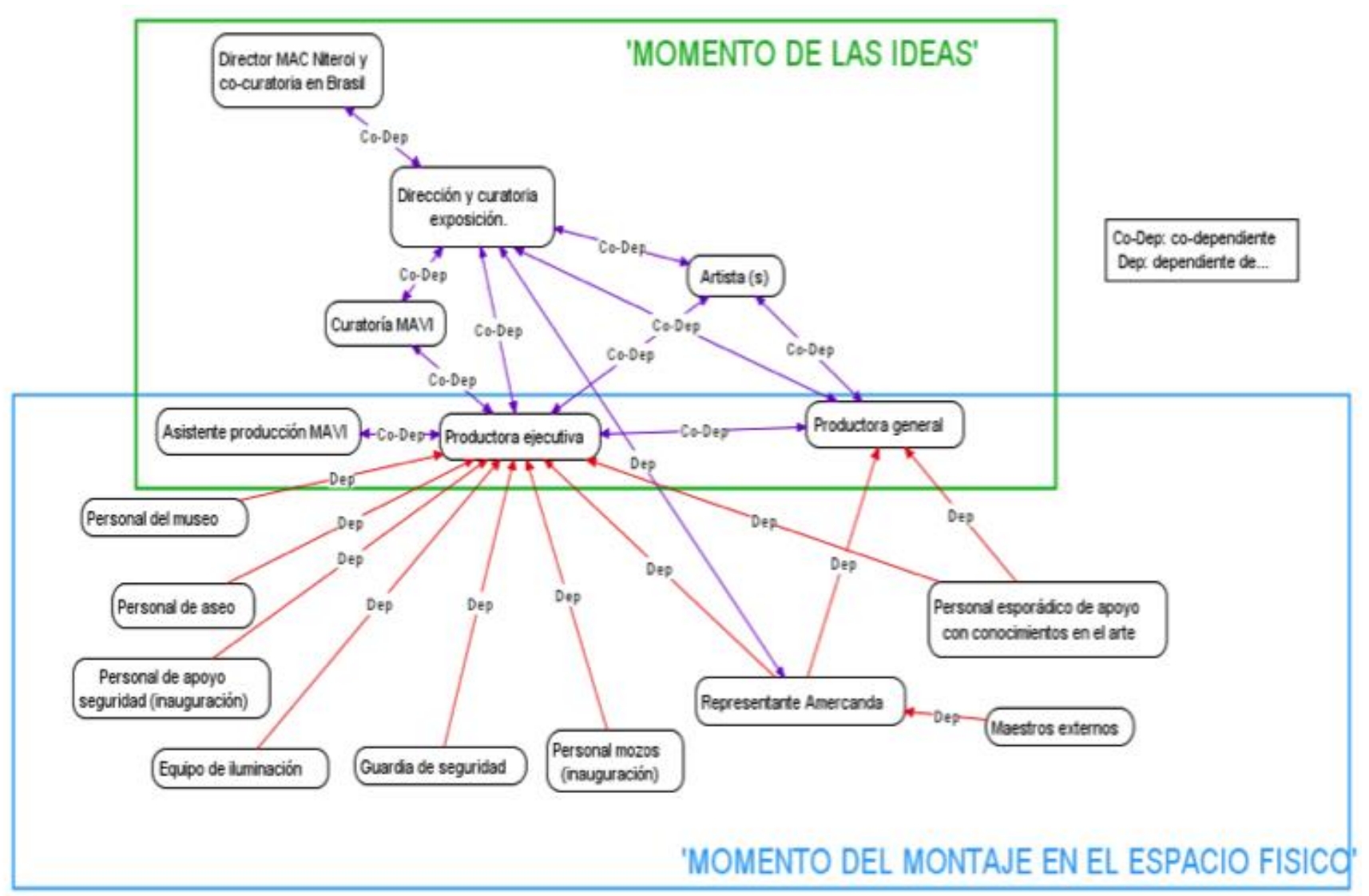


1 'Momento de las ideas' 3: Si tuviéramos que darles un orden temporal, sin duda alguna, este sería el primero de los tres. Ya que además de estar trabajando en el montaje mismo, este es un momento que parte desde mucho antes a trabajar en la exposición. Las ideas son aquí el foco.

Es cuando las personas más conocedoras de la historia y del sistema propio del arte producen el conocimiento artístico que luego será depositado en la audiencia. En palabras de Howard Becker estos serían los editores curatoriales, es decir, quienes transforman o buscan mantener el sentido de la obra. Este es el "grupo de personas que cooperan para producir cosas que por lo menos ellas llaman arte" (Becker,2005: 55), son los expertos sobre el mundo del arte que piensan la exposición. Son los que usan la "escritura estética [que es la que] insiste en una diferencia moral real entre lo que es arte y lo que no lo es" (Becker, 2005: 166). Decisión que está en manos de las distinciones que estos expertos trazan.
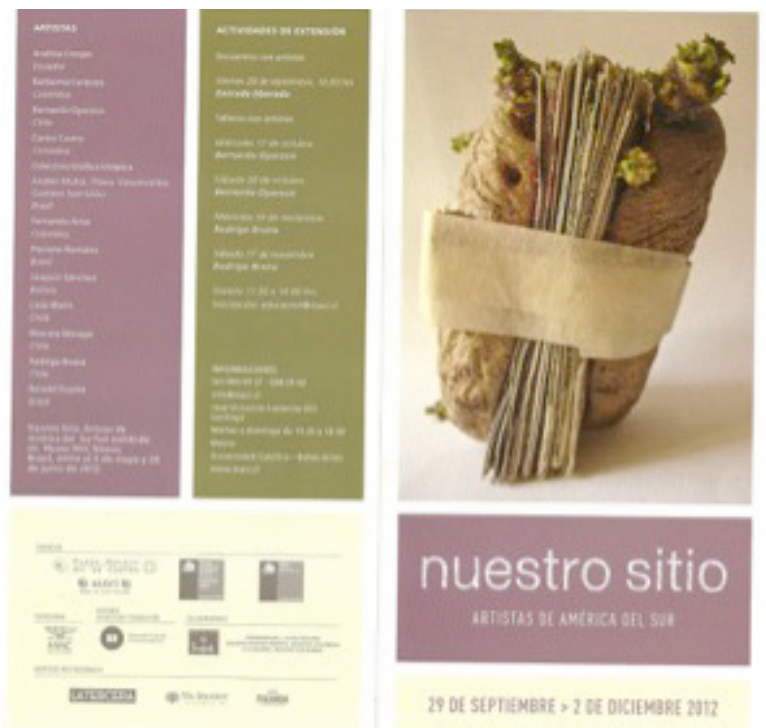

Folleto entregado a los asistentes de la exposición "Nuestro sitio".
Siguiendo la misma línea argumentativa, Ticio Escobar menciona que: "las creaciones populares no alcanzan ese grado superior, autocontemplativo y cerrado en sí que distingue las formas superiores del arte, y permanecen, por tanto, atrapadas por su propia materialidad, su técnica y sus funciones." (2008: 42). Resaltando la forma, como un concepto que tiene un rol de conformación en el arte.

Si revisamos una referencia en el catálogo de la exposición, escrito por la curadora de la exposición: "Nuestro sitio. Artistas de América del Sur, es una mirada particular que se aproxima desde la visualidad a ciertos acontecimientos y movimientos que se generan actualmente en nuestro continente" (Catalogo, 2012: 5), donde visualizamos una observación desde otro lugar.

Concretamente éstos son: partiendo por el artista(s), la dirección y curatoría de la exposición específica, curatoría del MAVI y por último, el director del MAC Niteroi y encargado a la vez de la co-curatoría de lo referente a Brasil. Son quienes generan "arte para sus interesados" (Espinosa, Ortiz, \& Moyano, 2012: 5).

Sobre la presencia de estos actores en la red, Isabel Tejeda dirá que abre una "controversia respecto a los papeles que el curador y el diseñador del montaje asumen, y que cuanto mayor ha sido la implicación de la obra con un espacio concreto en su instalación inicial, mayor competencia se exige a quien vuelve a montarla." (2006, 81). Coincidiendo que en este caso, la persona que adquiere mayor relevancia por su rol, es la curadora de la exposición. Es ella quien se encuentra a cargo de tomar el sentido de cada una de las obras de arte, pensarlas con otras de estas mismas, relacionarlos entre sí, y generar finalmente lo que todos vemos: la exposición "Nuestro sitio". Cumple la función de ser el nodo principal que une a todos los actores del momento de las ideas, y que además de coordinarlos entre ellos, trata con cada uno por separado, en especial esa relación se dio con los distintos artistas. 


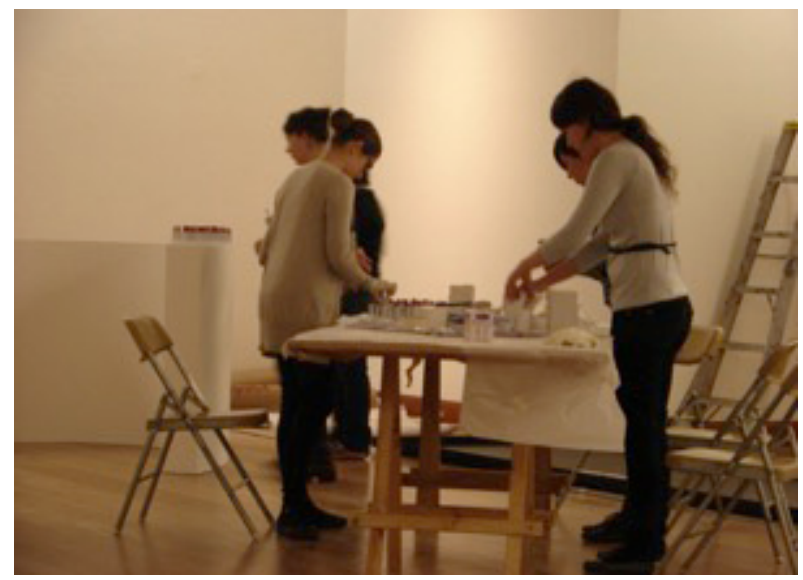

Personal de apoyo con conocimiento en el arte, montando una obra de arte.

2 'Momento del montaje en el espacio físico': $\mathrm{Si}$ el momento anterior se presentaba como el primero temporalmente, este podría considerarse el segundo (para comprenderlo mejor, ya que ambos se sobreponen). Es la puesta en práctica, en el espacio, de lo que se pensó que se podía hacer en el momento de las ideas.

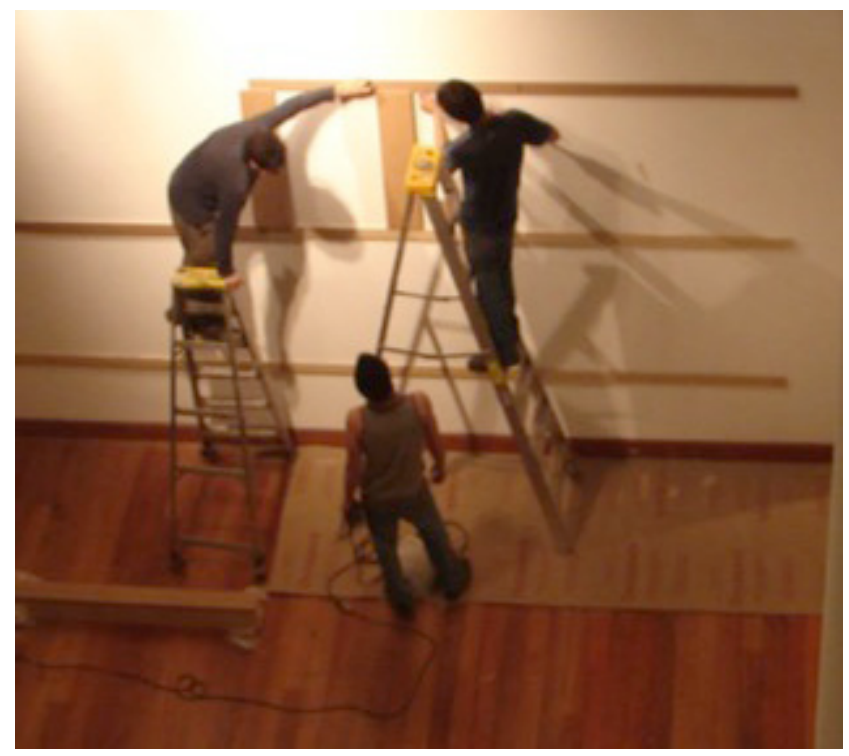

Maestros de compañía 'Amercanda'.

Aquí están variados tipos de personal: del museo mismo, de aseo, de apoyo en seguridad, mozos, maestros externos, equipo de iluminación y unos esporádicos con conocimientos en el arte. Éstos son los que menciona Becker como el personal de apoyo, es decir: "distintos grupos y subgrupos comparten el conocimiento de las convenciones de un medio, conocimiento que adquirieron de diversas maneras. (...) forman parte de la red cooperativa de la actividad que hace posible ese mundo y caracteriza su existencia" (2005: 88).

Lo impresionante es que, a pesar de no ser expertos del código del sistema del arte, aun así conocen en cierta medida algunas convenciones que deben respetar y que reproducen. Un ejemplo es el equipo de iluminación, compuesto por dos personas que durante los tres últimos días del montaje, avanzaban por toda la exposición, decidiendo (bajo la supervisión de curatoría y de producción) la forma precisa de iluminar las obras de arte. De hecho hubo varias conversaciones entre el equipo de iluminación y la curatoría y producción, donde la decisión era por parte de los últimos dos actores.

La autora Isabel Tejeda menciona la reconstrucción (o montaje físico) como parte del sentido de la obra, las que son "elegidas (...) por sus cualidades estéticas, táctiles y visuales" (Tejeda, 2006: 43), subrayando la categoría de la idea, en una posición por sobre el soporte material. Este es un cuestionamiento central ¿El sentido de la obra, entonces, también se ve influido por su montaje? Justamente lo que se busca aquí es dar cuenta de que los objetos forman parte de una realidad relacional que si se considerara en la comprensión de los fenómenos abocados al arte, posiblemente se podría lograr un análisis más amplio y complejo del fenómeno en

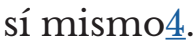

En este 'momento del montaje en el espacio físico', todos dependen en gran medida de la producción ejecutiva, y en menor medida, de la producción general. Quien se sale un poco de esa vinculación, es el representante de Amercanda (compañía externa y especializada en el arte, que fue contratada como apoyo en la construcción de la exposición), quien además se relacionaba de forma directa con la curadora de la exposición. Éste de hecho es el único nexo directo entre el 'momento de las ideas' y el 'momento del montaje en el espacio físico'; 
ya que como veremos a continuación, los demás actores de este tramo, dependen por completo del siguiente 'momento intermedio'.

3 'Momento intermedio': Este tramo es central en la configuración de la exposición. Es la unión y complementación entre el 'momento de las ideas' y el 'momento del montaje en el espacio físico'. Representado en el esquema con la superposición de los cuadros de los dos otros momentos de la red. Esta conexión que se produce es esencial, ya que finalmente es en donde se vela lo que los conocedores del arte quieren poner en práctica, pueda ser realmente concretizado por medio de las acciones del personal de apoyo.

Este momento es todo lo que conlleva la producción misma de la exposición. De hecho, está compuesto por la producción ejecutiva (del MAVI), la producción general (externa) y la asistente de producción (proveniente también del mismo museo).

Son los que llevan la cuenta económica, y van revisando el presupuesto (patrones de disponibilidad) constantemente para ver qué es posible de hacer. Están a cargo de los números, de la eficiencia y de que se logre llevar a cabo esta idea de exposición en el tiempo fijado. Es así, que "Todo patrón de disponibilidad refleja un tipo de organización social y se convierte en parte del patrón de limitaciones y posibilidades que conforma el arte que se produce" (Becker, 2005: 116), y lo que los artistas pueden llegar a hacer. Ya que el objetivo inmediato de este momento es la perpetuación del sentido de la obra, en concordancia con un espacio y una materialidad disponible específica, según los recursos económicos que dispone el museo.

La discusión sobre la relevancia de la existencia en un mismo espacio, de actores que actúen de 'puente' entre el primer 'momento de las ideas' y el segundo 'momento del montaje en el espacio físico", se ha tematizado en otros estudios. Un ejemplo es un estudio realizado en las galerías de arte contemporáneo de Francia, en que se concluye lo vulnerables que son éstas organizaciones ante la economía, y como ésta en su carácter performativo, no es muchas veces compatible con las decisiones estéticas (Benhamou, Moureau y SagotDuvauroux, 2002: 278). Esa incompatibilidad entre dos visiones en el MAVI es amortiguada por el "momento intermedio", resultando éste imprescindible para la operatividad misma del museo.

A lo largo de la etnografía, además de la existencia de estos tres momentos a lo largo del montaje, se vislumbró una nueva temática que no había sido considerada en el principio de la investigación: los dilemas.

\subsection{Dilemas en el montaje: diversos actores, diversos actantes, diversas visiones.}

Considerar a los actores no humanos, en relación a los humanos, es central: "Al compartir la trascendencia con los objetos y al acceder a ellos por los mil conductos del lenguaje, de la práctica y de la vida social, ya no estamos obligados a reducir las circunstancias, sea a la naturaleza, sea a la sociedad, sea al discurso" (Latour, 1994:106). Dehecho, durante el transcurso de la aproximación etnográfica, al ser considerada esta postura teórica fue que emergieron distintas situaciones que llamaré dilemas: instancias o conversaciones cruciales que poseen entre los actores visiones diversas sobre algo en común, en las que se necesita llegar a cierto consenso mínimo, donde adquiere una gran relevancia el espacio arquitectónico del MAVI.

Son problemáticas que se vivieron muchas veces a lo largo del montaje, y fueron registradas en la etnografía. De hecho conforme se acercaba la fecha de la inauguración, éstas tendían a ser entendidas como tensiones en la toma de ciertas decisiones. A veces tuvieron una relevancia menor, pero también muchas fueron conversaciones que duraban un largo tiempo. A continuación describiré algunas de los dilemas del montaje que resultan más llamativos a la luz de ciertas lecturas. 


\section{1. ¿Tarugo o clavo?}

Es el tercer día en el montaje de la exposición, y la artista, la curadora, un maestro del museo y una asistente especializada en arte, buscan tomar una decisión: Adherir las tazas que se quieren colocar en la pared, las que en su conjunto son conocidas como la obra de arte 6. Así, los primeros tres tienen opiniones totalmente distintas, aunque la mayor diferencia pareciera radicar entre la curadora con la artista, versus el maestro. Las primeras querían afirmar con clavos, buscando la preponderancia del sentido de la obra y de la perpetuación de la belleza de ésta. Mientras que el segundo actor, se enfoca en la utilidad del objeto, ya que usando el tarugo, es imposible que se puedan caer las tazas.

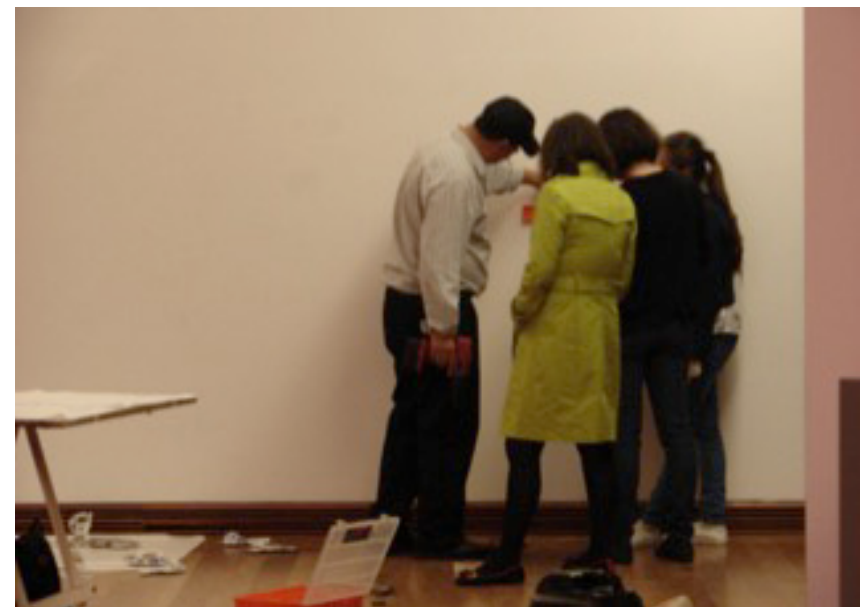

Maestro, curadora, artista, personal de apoyo revisando y discutiendo del montaje de la obra.

Cuando se coloca en práctica el plan elaborado con anticipación, es que se develan este tipo de dilemas. Es "Sólo mediante la amplificación del proceso de generación de pequeñas diferencias en la fabricación de objetos, uso y ajuste, una instalación técnica nueva puede emerger" (Yaneva, 2003: 186). Así la obra de arte, es influida por la forma en la que se presenta y según el espacio en el que se encuentra.

En el arte contemporáneo, los "museos pasan de la conceptualización y realización de conocimiento conformado por las antiguas formas e imágenes, al compromiso del objeto como un complejo campo de la traducción." (Cameron \& Mengler, 2009: 215). Lo que antes de instalar la obra eran solo objetos (tarugos y clavos), ahora son parte fundamental de la conformación de ésta. De hecho sucede que la decisión, si va un tarugo o un clavo, pasa a formar parte de las distinciones que operan sobre la gestación de la obra en la exposición.

\section{2. ¿Se diferencian las obras de artes y/o los espacios?}

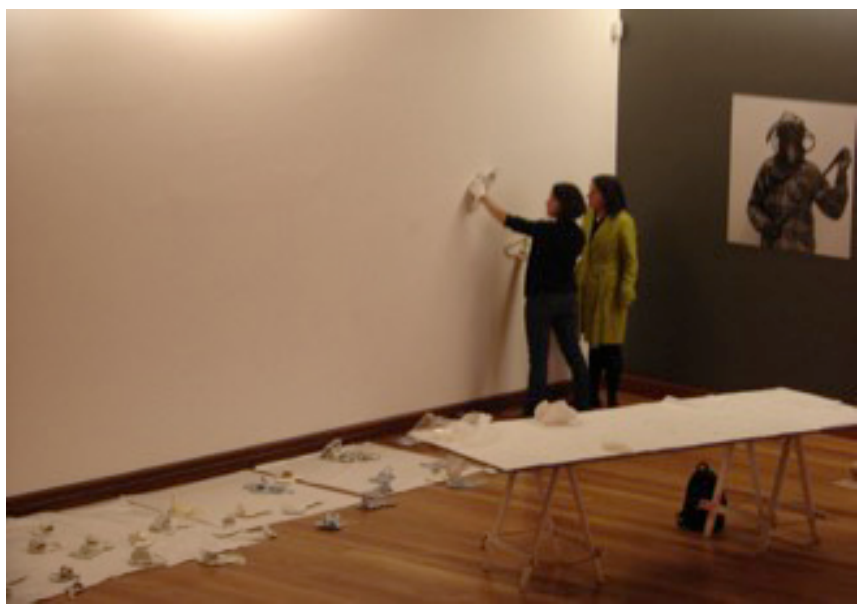

Artista y curadora discutiendo sobre la distribución en el espacio del montaje.

El primer día del montaje, la preocupación principal era la pintura. Los actores del 'momento de las ideas' se preguntaban ¿Eran los colores indicados? ¿El color hace resaltar las obras que irán en esos lugares? ¿Se diferencian entre sí las distintas obras de la exposición? ¿Ayuda la pintura en ello? Mientras que los del 'momento intermedio' querían saber si es que era necesario tener que ir a comprar más, pensando que había que volver a comprar un color muy específico. Pero también estaban los del 'momento de montaje físico', que pensaban si había suficiente pintura y cuánto tiempo más estarían trabajando en las murallas del museo, para pasar a la siguiente etapa.

$Y$ es que el espacio es tan relevante en la construcción de una exposición, que de ello depende que se logre plasmar el sentido de la obra. Entonces, además de la influencia de los diversos actores, hay que tomar en cuenta la trayectoria de cada uno de estos para llegar a como son usados: "No únicamente los objetos, si no la trayectoria de sus usos. Apreciaríamos 
que la disposición de las salas incite a ver y a criticar lo que ciertos poderes vieron, a crear otros modos de inventariar y catalogar para entendernos con los diferentes." (Castilla, 2010: 143) Si las murallas del museo no hubieran sido pintadas con distintos colores, quizás no hubiera sido posible la fácil diferenciación por parte del público de las obras. Y menos, el acceso de parte de los presentes, al sentido que en el 'momento de las ideas' se buscaba plasmar en la exposición.

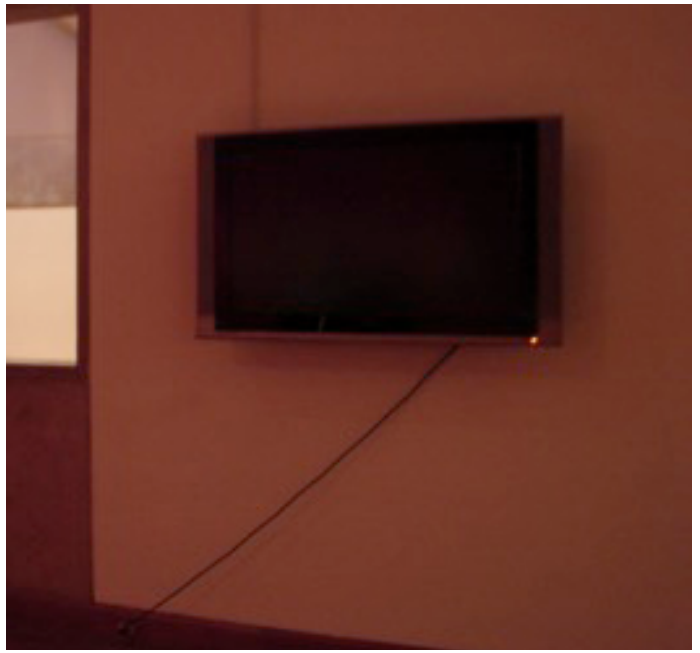

Televisor instalado en la inauguración.

Una conversación entre la artista y la curadora llama la atención: "¿Se nota la diferencia verdad?" "si, si, es que ese gris te los separa totalmente, es que se ve bellísimo, se ve muy bien, bien". La relevancia que adquieren las obras en sí mismas, se refuerza únicamente en el espacio en que son insertadas. La diferencia tan mínima quizás, de que fueran distintos colores según la muralla y la obra, resulta ser de esencial importancia para los que componen el 'momento de las ideas'. Quienes, buscando mantener el sentido, hacen ver la necesidad de diferenciar las obras de arte entre sí, por medio del uso correcto de los espacios del museo. Tal como se escuchó en el montaje: "está como potente, como fashion, así el museo, es una obra en sí mima este museo, este espacio". Solo la diferenciación en el espacio, provoca la diferenciación entre las obras de arte, que con lleva la perpetuación del sentido de estas mismas.

\section{3. ¿10 centímetros más a la derecha?}

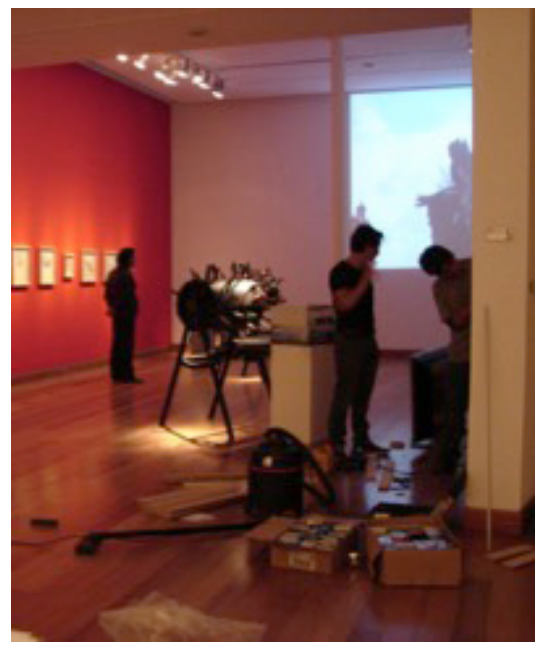

Maestros revisando como instalar el televisor.

En una sala, donde se encontraba ya montada una obra y un video (que era proyectado en una de las murallas), en un costado el tercer día se instalaba un televisor negro de pantalla plana. Éste se fijó por medio de un complicado procedimiento a la muralla con ayuda de varios tarugos, unos soportes de madera (que fueron hechos para esta ocasión y ese televisor) y los maestros de Amercanda. En el televisor se proyecta la obra "Fuego cruzado" de Ronald Duarte. Cuando hace su aparición la curadora y la producción de la exposición a supervisar como quedó fijado, inmediatamente se percatan de algo: "Me parece que está bien... ¿Por qué no está conectado al enchufe?" A lo que un maestro responde, que ese era justamente el problema: el cable (que era parte del aparato e irremplazable) de la televisión no alcanzaba a ser enchufado, por lo que tenía que ser movida solo 10 centímetros más a la derecha o el uso de un alargador, pero que no afectaría mucho. Pero para la visión proveniente del 'momento de las ideas' esto si producía cambios estéticos, y comienza a ver otras opciones posibles para que el plan que ya se había pensado antes, fuera llevado a cabo. Así la producción responde de forma de puente entre ambos, viendo la viabilidad concreta de ambas partes. Finalmente se decide que se debe mover 10 centímetros hacia la derecha toda la instalación, porque sin la electricidad, la obra no se exponía. 
Solo este episodio, les llevó una conversación de por lo menos una hora para ser resuelto. Hay tres visiones marcadas y diferenciadas entre sí, donde cada uno de los actores quiere hacer cumplir su rol en relación con el objeto.

Desde la visión de Yaneva, "En una instalación de objetos de arte al empezar a desviarlos de sus funciones "estándares" y más numerosas variaciones se producen materiales de diferentes velocidades." (2003: 184). La velocidad y la comprensión de la obra misma son transformadas desde su idea original, a una nueva. Es la materialidad de la obra expuesta, la que produce una diferencia en la composición de la obra de arte.

En cuanto a "Las instalaciones existentes (salas de concierto, galerías y museos de arte, y bibliotecas) tienen una cantidad finita de espacio, las pautas de gusto limitan el uso que puede darse a ese espacio" (Becker, 2005: 171). Es decir, la arquitectura toma un valor central en la conformación de la obra.

Manuel Tironi por su parte, concluye en su artículo "Nuestros resultados indican que la localidad se realiza a través de las prácticas de los miembros de la escena. Pero también muestran que, a su vez, los miembros y sus prácticas son un efecto de la espacialidad particular en el que expusieron sus redes productivas" (2012: 206) Se suma a la relevancia de la arquitectura la localidad, ya que ésta se relaciona con los actores, y éstos a su vez con la localidad misma, por lo que hay que recordar que el Museo de Artes Visuales se encuentra ubicado en el centro de Santiago de Chile, en un conocido circuito turístico. Por lo tanto la arquitectura y la localidad (la Plaza Mulato Gil de Castro) son elementos que se ven influidos y que influyen en la construcción de una exposición de arte contemporáneo en el MAVI. La Plaza pasa así a ser un espacio clásico y central para la construcción de escenas de avanzadas en Santiago de Chile.

\section{4. ¿Cuál es el orden indicado para los cojines?}

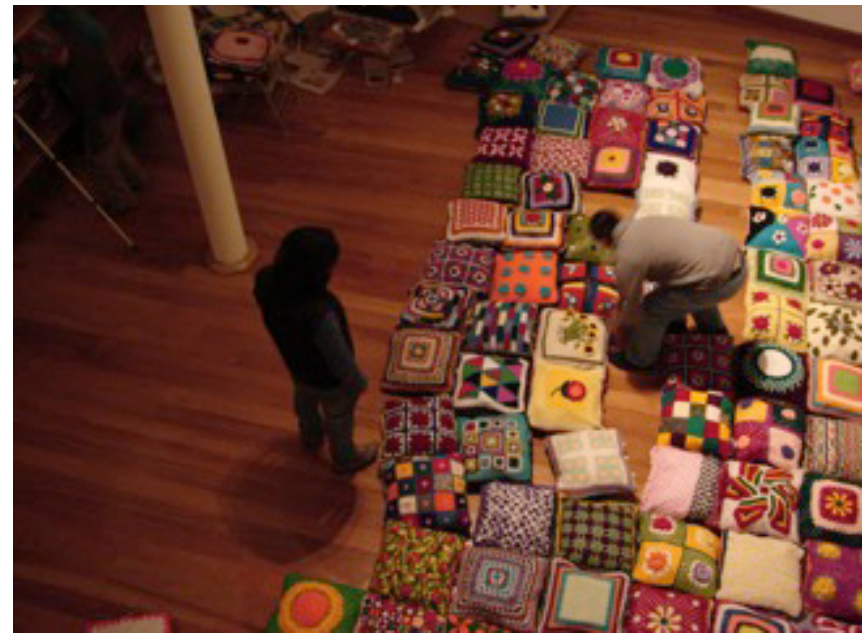

Artista y su asistente montando la obra.

La "producción artística es una forma material en la práctica, que emerge y se desarrolla a través del material comprometido con el mundo" (Domínguez, 2012: 152). Existe así, todo un mundo que emerge en la misma práctica del montaje de los objetos conocidos como obra. Un ejemplo de ello, es el que viene a continuación.

En dos días, diversos asistentes que ayudan en el montaje, fueron rellenando con papel de diario varios cojines tejidos. En paralelo, los maestros de Amercanda trabajan colocando una gran estructura de paneles en donde se atornillan desde la base floreros con flores de plástico en posición horizontal. Entonces aparece el artista de la obra con dos asistentes (estudiantes universitarios de arte). Se acerca a los cojines, y los asistentes los van colocando simétricamente al frente de la estructura con los floreros en la pared. A medida que realizan este trabajo, el artista permanece al lado observando. Los asistentes van y vienen con los cojines, cambiándolos de un lugar a otro, varias veces, siempre cuidando mantener un rectángulo imaginario rodeando a todos. El artista en un momento se inserta con los asistentes y cambia algunas cosas. Este escenario, sucede por lo menos durante dos horas. Finalmente la obra queda casi lista, al otro día afinan unos detalles. 
$\mathrm{Al}$ acercarme a uno de los asistentes le pregunto: "¿Por qué los cojines van en cierta posición y porque no en otra?" a lo que él responde "Por simetría, que hay que hacerla lo más simétrico posible para... por ejemplo tenemos que colocarlos para que no queden solo en un lugar los con más colores o los con una forma cuadrada dentro", "¿Pero entonces siempre queda distinta la obra?" "Em... si, en la composición, de forma mínima, lo que importa, el contenido, el sentido, es el mismo".

En este caso vemos cómo el rol que cumple la observación es central, de hecho que actor mira y describe la obra, influye en la comprensión de la misma: "El significado de un objeto varía no sólo con el tiempo y el espacio, sino también en función de quién lo estaba viendo." (Alberti, 2005: 568). Niklas Luhmann por su parte también hace referencia a ello: "Todo trato con el arte es en primer lugar una observación que distingue: también y sobre todo el proceso en el que se realiza la gestación de la obra. El artista toma en ello parte como observador (...) la forma debe ser capaz de conducir la observación" (1999: 30). Entonces la posición desde la que observa el asistente del artista y lo que luego observará un asistente a la exposición (dependiendo de su inserción en el sistema del arte) influye sobre el sentido del objeto llamado obra.

\section{Conclusión}

Entonces ¿Qué pasa con la obra de arte? ¿Cuándo nace? Distintos autores que ya revisamos aportan algunas luces sobre el tema. Para Luhmann la obra de arte es una multiplicidad de distinciones (25); Howard Becker aporta con su visión en torno a la cooperación de diversos actores y no solo del artista; Yaneva señala que los objetos de arte nacen por una profusión horizontal, por la vibración y la extensión del tejido denso de las diferencias (2003: 183); y por último, según Domínguez, la obra existe siendo un logro contingente de materialidades que emergen desde una producción y un sitio específico (2012: 157). Una propuesta reciente ha comenzado a ser explorada por Antonine Hennion (9), quien propone los estudios críticos en la sociología del arte como una transformación de la investigación que se ha llevado a cabo hasta ahora, donde en el análisis social los mediadores juegan un rol central a ser considerado en "el análisis de lo institucional, material, [y en los] intermediarios humanos" (Hennion y Grenier, 2000: 347), realizando un intento por rescatar la relevancia de la obra de arte.

Esta investigación se enmarca en un enfoque alternativo en línea con algunos de los autores anteriormente señalados, de tal forma de ampliar el foco investigativo en la sociología del arte, indagando en varias líneas que emergieron en el transcurso de la etnografía: las etapas que conlleva la conformación de una exposición en un museo; la profundización de lo que se entiende por un montaje, considerando las redes que lo componen; y la emergencia de dilemas que influyen en la obra de arte, lo cual, en complementación con la teoría mencionada, es que se concluye que la gestación de la obra de arte contemporáneo no es un proceso que emerge únicamente del artista, sino que emerge cuando estéticamente se presenta el resultado de un trabajo cooperativo de variados actores (humanos y no humanos) en un espacio con una forma previamente determinada y por distinciones propias del lenguaje artístico.

Sin duda alguna, este es un tema que requiere una mayor profundización, ya que podemos decir que la definición se aplica correctamente con la información recolectada a partir de este caso en específico. ¿Pero qué sucedería en otro caso? ¿Solo se aplica este fenómeno en el arte contemporáneo? ¿Cuál es la narración de los actores involucrados en relación con los objetos? Estas son solo algunas de las interrogantes que en adelante pueden dar cabida a nuevas investigaciones, y no solo en la sociología sobre la gestación de la obra de arte. Sin duda alguna, se considera que para responder a estas preguntas y hacerse cargo de ellas, cualquier propuesta tendría que considerar el punto de vista y las implicancias que tiene el método utilizado en estas páginas con respecto al tema de estudio escogido, es decir, desde la etnografía y la sociología del arte, en relación con la noción de obra en construcción en el montaje de una exposición, tema que puede abrir más líneas de posibles investigaciones a futuro.

Las distintas visiones de la sociología del arte, sintetizadas por Nathalie Heinich, siempre están en distinción con lo que se entiende por 'la sociedad', cuando resume esta sociología en tres generaciones: 'arte y sociedad', 'arte en la sociedad' y 'arte como 
sociedad' (2002: 16-17). Pero en realidad no hay un cuestionamiento por lo que entienden por esta última ¿Solo la sociedad de personas? La sociedad que envuelve a la obra ya no son solo las personas del sistema del arte, sino que se deben considerar los objetos y los espacios que influyen en el proceso de construcción de la obra como parte de las comunicaciones del arte. No obstante, la gestación no se da por las cualidades intrínsecas del artista, sino que la obra en la sociedad es un proceso continuo de ensamblajes cotidianos entre cooperaciones de actores humanos y no humanos que gatillan comunicaciones en su contingencia (Ortiz, 2014: 45). A diferencia de Heinich, aquí se ha postulado que los actores no humanos si influyen en la obra de arte ¿Por qué entonces no pensar una nueva generación?

\section{Bibliografía}

Acord, S. (2010): "Beyond the Head: The Practical Work of Curating Contemporary Art". Qualitative Sociology, 33 , pp. 447-67.

Alberti, S. (2005): "Objects and the Museum. The history of Science Society”. Isis, 96, pp. 559-71.

Álvarez-Gayou, J.L. (2009): Como hacer investigación cualitativa. Fundamentos y metodología, México, Paidós.

Banks, M. (2010): "Introducción", en Los datos visuales en la investigación cualitativa, Madrid, Morata.

Becker, H. (2005): Los mundos del arte, Buenos Aires, Universidad Nacional de Quilmes.

Becker, H. (2011): Trucos del oficio, Buenos Aires, Siglo Veintiuno Editores.

Benhamou, F., Moureau, N. y D. Sagot-Duvauroux. (2002): "Opening the Black Box of the White Cube: a suvey of French contemporary art galleries at the turn of the millennium", Poetics, 30, pp. 263-80.

Bourdieu, P. (2002): Las reglas del arte. Génesis y estructura del campo literario, Barcelona, Anagrama.

Bourdieu, P. (2010): El sentido social del gusto. Elementos para una sociología de la cultura, Buenos Aires, Siglo Veintiuno Editores.

Cameron, F. y S. Mengler. (2009): "Emergent Metaphors for a Complex World Complexity, Transdisciplinarity and Museum Collections Documentation", Journal of Material Culture, 14, pp. 189-218.
Castilla, A. (2010): El museo en escena. Política y cultura en América Latina, Buenos Aires Paidós.

Catálogo. (2012): Nuestro sitio. Artistas de América del Sur, Santiago de Chile, Museo de Artes Visuales y MAC de Niteroi.

Dominguez, F. (2012): "The material production of the Spiral Jetty: A study of culture in the making", Cultural Sociology, 6, pp. 143-61.

Escobar, T. (2008): El mito del arte y el mito del pueblo, Santiago de Chile, Metales pesados.

Espinosa, A., Ortiz, F., y C. Moyano. (2012): "Exclusión e Inclusión en un Museo de Arte Contemporáneo: Fundación Cultural Plaza Mulato Gil de Castro", Ponencia, Universidad de Chile.

Geertz, C. (1992): La interpretación de las culturas, Barcelona, Gedisa.

Goffman, E. (2004): La presentación de la persona en la vida cotidiana, Buenos Aires, Amorrortu.

Gúber, R. (2001): La etnografía, método, campo y reflexividad, Bogotá, Grupo Editorial Norma.

Hammersley, M. y P. Atkinson. (2001): Etnografía: métodos de investigación, Barcelona, Paidós.

Heinich, N. (2002): La sociología del arte, Buenos Aires, Nueva Visión.

Hennion, A. y L. Grenier. (2000) Sociology of Arte: New Stakes in a Post-Critical Time. Eds. Stella Quaj y Arnauld Sales, UK, SAGE Publications.

Hennion, A. (2004): Pragmatics of Taste. Eds. Mark Jacobs y Nancy Hanrahan, Oxford, Blackwell.

Latour, B. (1994): "Etnografía de un caso de "alta tecnología”: Sobre Aramis". Política y Sociedad, 14-15, pp. 77-97.

Latour, B. (1994) “'Tienen historia los objetos? El encuentro de Pasteur y de Whitehead en un baño de ácido láctico", Vrin, 2, pp. 197-217.

Latour, B. (2001): La esperanza de Pandora, Barcelona, Gedisa.

Law, J. (2007): After method. Mess in social science research, London, Routledge.

Luhmann, N. (1999): Teoría de los sistemas sociales II (artículos), Barcelona, Universidad Iberoamericana.

Ortiz, F. (2014): “Book Review: “¿Es posible una cuarta generación en la sociología del arte? Una reseña de "La sociología del arte" de Nathalie Heinich", Doble Vínculo, 5, pp. 42-45. 
Ramos, C. (2012): El ensamblaje de ciencia social y sociedad, Chile, Universidad Alberto Hurtado Ediciones.

Taylor, S. J., y R. Bogdan. (2000): Introducción a los métodos cualitativos de investigación, Buenos Aires, Paidós.

Tejeda, I. (2006): El montaje expositivo como traducción, Madrid, Trama editorial.

Tironi, M. (2012): "Enacting Music Scenes: Mobility, Locality and Cultural Production”, Mobilities, 7, pp. 185210.

Wolcot, H. (2006): "Etnografía sin remordimientos", Revista de Antropología Social, 16, pp. 279-96.

Yaneva, A. (2003): "Chalk steps on the museum floor: The 'pulses' of objects in an art installation", Journal of Material Culture, 8, 169-88.

Yaneva, A. (2003): "When a bus met a museum: following artist, curators and workers in art installation”, Museum and Society, 1, pp. 116-31.

Agradecimientos: Agradezco al Museo de Artes Visuales por las facilidades y el interés mostrado en esta investigación, especialmente a Beatriz Salinas e Irene Alcalde. Además, agradezco enormemente por los comentarios, las sutilezas que observaron y el apoyo constante de Fernando Valenzuela como profesor guía de esta tesis, y a Alejandro Espinosa, por sus constantes aportes a este documento.

Recibido: 14 de abril. Aceptado: 6 de julio de 2017.

\section{Notas}

1 La fotografía fue tomada por la autora del artículo, en el mismo transcurso de la aproximación etnográfica (entre los días lunes 24 y el viernes 28 de septiembre del 2012). Todas las fotografías publicadas en este artículo tienen el mismo origen, y fueron autorizadas previamente por el Museo de Artes Visuales para ser publicadas.

2 Frase que utilizaron varios de los actores con quienes se conversó en el montaje de la exposición para describir al MAVI.
$3 \quad$ Los nombres de los momentos derivaron de expresiones que ocupaban algunos de los actores que componen la red del MAVI. De hecho estos nombres fueron corroborados luego de que se les mostrara a algunos de los actores directivos del curso.

$4 \quad$ Esta realidad relacional a la que se hace alusión, es un análisis complejo y profundo del que este artículo no logra hacerse cargo del todo, debido a la extensión. Aun así se considera que cuando se piensa desde una perspectiva relacional, se entiende que conjuga más conceptos, que produce y reproduce un modelo de sociedad, en tanto la exposición de arte se co-contruye con un contexto social y político determinado. Estos antecedentes no son considerados para el desarrollo del artículo, para centrarse en desarrollar la idea en torno a la definición de obra de arte y su cuestión, a pesar de lo cual si se admite que su consideración en la investigación más amplia fue tomada en cuenta.

$5 \quad$ Referencia obtenida de una de las conversaciones con la producción del Museo de Artes Visuales buscando autodefinirse.

6 Comprendiendo que la obra de arte es un objeto en sí mismo en construcción. Para posibles definiciones de esta, se recomienda la lectura de los siguientes textos: Heinich (2002), Yaneva (2003), Becker (2005) y Alberti, S. (2005). 